EPJ Web of Conferences 70, 00070 (2014)

DOI: $10.1051 /$ epjconf/ 20147000070

(C) Owned by the authors, published by EDP Sciences, 2014

\title{
The neutrino telescope ANTARES
}

\author{
Andreas Gleixner ${ }^{\mathrm{a}}$ on behalf of the ANTARES collaboration \\ ${ }^{1}$ Friedrich-Alexander-Universität Erlangen-Nürnberg \\ Erwin-Rommel-Str.1, 91058 Erlangen \\ Germany
}

\begin{abstract}
The ANTARES neutrino telescope is currently the largest neutrino detector in the Northern Hemisphere. The detector consists of a three-dimensional array of 885 photomultiplier tubes, distributed along 12 lines, located at a depth of $2500 \mathrm{~m}$ in the Mediterranean Sea. The purpose of the experiment is the detection of high-energy cosmic neutrinos. The detection principle is based on the observation of Cherenkov-Light emitted by muons resulting from charged-current interactions of muon neutrinos in the vicinity of the detection volume. The main scientific targets of ANTARES include the search for astrophysical neutrino point sources, the measurement of the diffuse neutrino flux and the indirect search for dark matter.
\end{abstract}

\section{Introduction}

Evidence for the existence of high-energy cosmic rays has been existing for about a century. While there are many candidates for galactic and extragalactic accelerators of cosmic rays - e.g. supernova remnants, micro quasars, active galactic nuclei or gamma ray bursts - the origin of cosmic rays remains unknown. The observation of cosmic neutrinos could help to identify the sources and, since only the measurement of cosmic neutrinos would be an unambiguous signature of hadronic acceleration, understand the acceleration mechanisms of cosmic rays.

There are some other distinct advantages of using neutrinos as messenger particles. Neutrinos are only interacting weakly, so they are not affected by the inter- and extragalactic magnetic fields. Consequently their trajectories are pointing back to their origin, in contrast to the bulk of cosmic rays, which consists of charged particles. Neutrinos can pass unhindered trough dense accumulations of matter, making them suitable for observations not possible with traditional means of astronomy. Neutrino signals can be time correlated with optical signals.

Also the search for astrophysical neutrino point sources is the main scientific target for large neutrino telescopes like ANTARES, such telescopes are multi-purpose devices. Further scientific targets of ANTARES include, but are not limited to, the measurement of the diffuse neutrino flux, the indirect search for dark matter and the search for magnetic monopoles.

a e-mail: andreas.gleixner@physik.uni-erlangen.de

This is an Open Access article distributed under the terms of the Creative Commons Attribution License 2.0, which permits unrestricted use, distribution, and reproduction in any medium, provided the original work is properly cited. 


\section{The ANTARES neutrino telescope}

The detection principle of the ANTARES[1] neutrino telescope relies on the detection of CherenkovLight, emitted by high-energy charged particles originating from charged current neutrino interactions in the vicinity of the detector. The main detection channel consists of charged current interactions of high-energy muon (anti-)neutrinos:

$$
v_{\mu}+N \rightarrow \mu+X
$$

The high-energy muon, produced in this interaction, has an energy-dependent path length up to several kilometers. Its trajectory is pointing roughly in the same direction as the trajectory of the neutrino, the average angle between both trajectories decreases with energy. The Cherenkov-Light is detected with a three dimensional array of photomultiplier tubes (PMTs). The trajectory of the muon can be reconstructed using the arrival times and the amplitudes of the Cherenkov-Photons emitted by the muon and registered by the PMTs (hits). The track reconstruction is usually performed with a maximum-likelihood estimation, based on a probability density function for the photon arrival times. The energy of the neutrino can be estimated with the observed energy loss of the muon in the detector.

The ANTARES detector consists of a three dimensional array of 885 optical modules (OMs) in the Mediterranean Sea, near the French Coast. Each OM houses a 10" PMT in a pressure resistant 17" glass sphere. The OMs are looking downward at an angle of $45^{\circ}$ for the optimized observation of up-going muons (due to the large amount of atmospheric muons which penetrate the water, usually only muons reconstructed as up-going are used for analysis). Three OMs form a storey, which also houses electronics for readout and calibration. 25 stories, connected with a flexible cable and kept taut by a buoy at the top, form a line. The 12 lines of the ANTARES detector are anchored at the sea-floor in a depth of $2475 \mathrm{~m}$ with a separation of about $74 \mathrm{~m}$. The detector covers an area of $180 \times 180 \mathrm{~m}^{2}$ on the sea-floor, the height of one line is about $450 \mathrm{~m}$. See fig 1 . Since the lines can move in the sea current and the storeys can rotate around the line axis, it is necessary to observe the position and orientation of each OM. For this purpose, a system of acoustic emitters and receivers and a system of compass and tiltmeters is used. With the acoustic system, the relative positions of 5 storeys per line are calculated. With the compass and tiltmeters, the orientations of all storeys are measured. A physical model of the lines moving in the sea is used to fit the alignment of the detector to the data. With this method, the positions of the OMs can be calculated with a precision better than $10 \mathrm{~cm}[2]$.

All raw data is transmitted to a shore station with an optical cable with a length of about 40 $\mathrm{km}$. At the shore station, filters are applied before the data is permanently stored. The requirements for an event to be triggered are usually based on the occurrence of hits with either a high charge (several photo-electrons) or coincident hits (hits on several OMs of one storey within a certain time window). While this hit-signatures are typical for the Cherenkov-Light produced by high-energy muons, the optical background consists mainly of uncorrelated single-photon hits. Filtering the raw data is necessary due to the large amount of optical background (see next section). The first data was taken in Januar 2007 (ANTARES has been continuously taking data since then), the detector has been completed in May 2008.

\subsection{Background}

There are two main sources of background for the detection of cosmic neutrinos. Down-going atmospheric muons (muons originating in cosmic ray induced air showers), which have passed trough the approximately $2 \mathrm{~km}$ of seawater above the detector, can be falsely reconstructed as up-going. The atmospheric muon rate is usually very high compared to an expected signal, so the suppression of this background is highly important. This can be done with cuts on the quality parameters of the track 


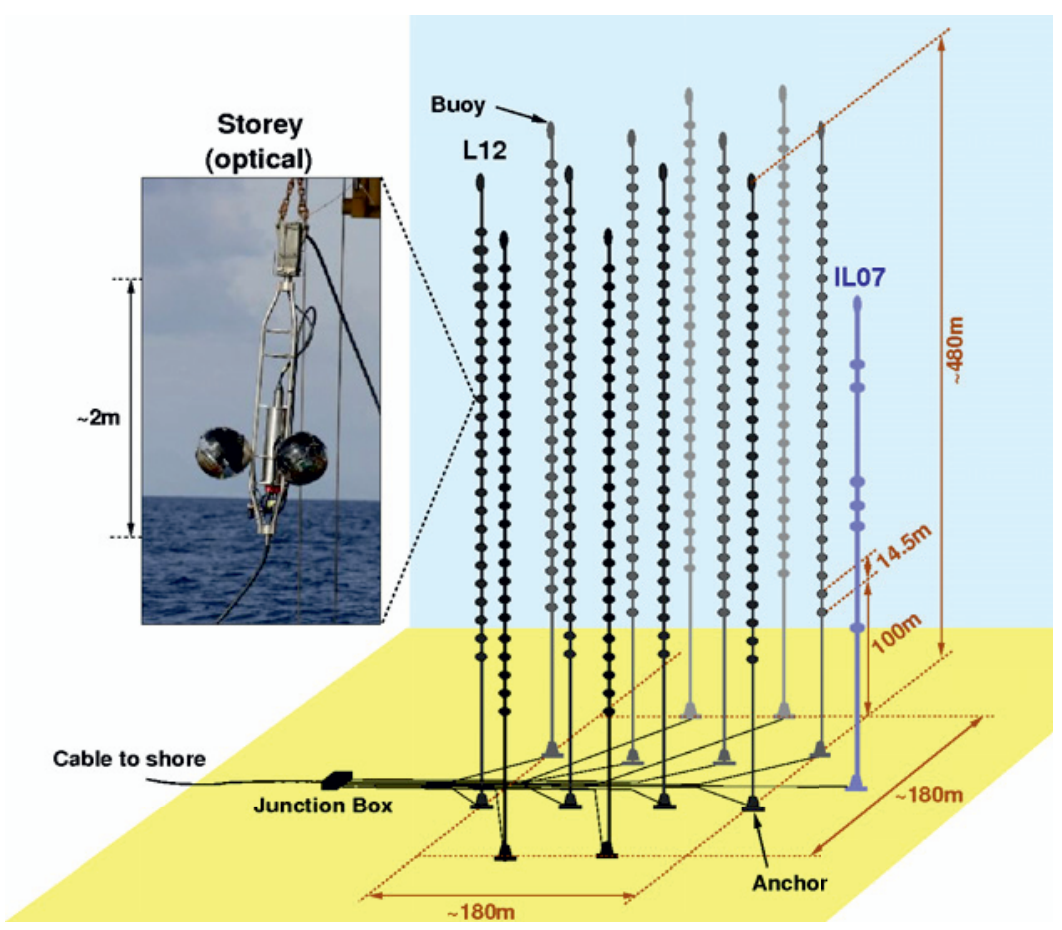

Figure 1. Schematic view of the ANTARES detector.

reconstruction algorithms or additional filters. The second source consists of atmospheric neutrinos originating in cosmic ray induced air showers in the opposite hemisphere. The rate of muons induced by atmospheric neutrinos is lower than the atmospheric muon rate by orders of magnitude. Depending on the analysis, the atmospheric neutrino background can be reduced by cuts on parameters depending on the energy of the muon.

The Cherenkov-Light emitted by relativistic electrons originating from the beta-decay of Potassium-40 and bioluminescence are responsible for an optical background. Typical rates per PMT are between $60-120 \mathrm{kHz}$ with additional short bursts and periods with higher rates. This background is locally dominat over the background from atmospheric neutrinos and muons[3].

\section{Search for neutrino point sources}

For this analysis, data from 29.01.2007 to 14.11.2010 was used, with a total of 813 days of data taking. There were two different approaches for the neutrino point source search, a full sky search and a candidate list search for 51 selected potential neutrino sources.

\subsection{Event Selection}

To find optimal event selection criteria, Monte-Carlo simulations were used. The atmospheric neutrino background was weighted according to the Bartol[4] parameterization, the atmospheric muon background was simulated with the MUPAGE[5] package. Two cuts were used in this analysis. First, 

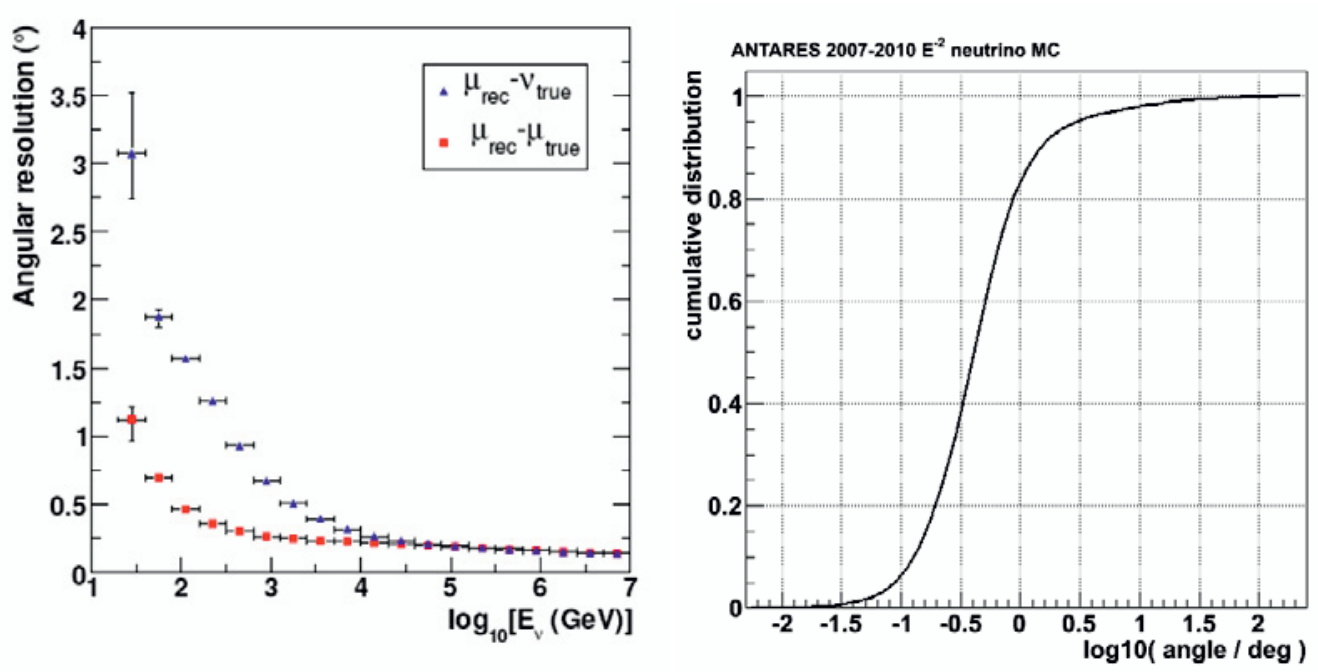

Figure 2. Left plot: Energy dependent distribution of the median angle between the reconstructed muon and the simulated muon (red) and the simulated neutrino (blue). The average angle between the trajectories of muon and neutrino decreases with energy. Right plot: Integrated distribution of the angle between the reconstructed muon and the neutrino for events that pass the selection cuts. $50 \%$ of those events are reconstructed better than $0.46^{\circ}$. An $\mathrm{E}^{-2}$ spectrum was assumed.

Table 1. Effect of selection cuts on the number of events for data (second column), simulated atmospheric muons (third column), simulated atmospheric neutrinos (fourth column) and a simulated $\mathrm{E}^{-2}$ neutrino signal (fifth column). [6]

\begin{tabular}{l|l|l|l|l} 
& data & atm. $\mu$ & atm. $v$ & $\mathrm{E}_{v}^{-2} v$ \\
\hline Triggered events & $3.94 \times 10^{8}$ & $3.06 \times 10^{8}$ & $1.54 \times 10^{4}$ & $100 \%$ \\
\hline Reco. upgoing events & $6.08 \times 10^{7}$ & $2.98 \times 10^{7}$ & $1.24 \times 10^{4}$ & $61 \%$ \\
\hline Reco. upgoing events $+\beta<1^{\circ}$ & $3.90 \times 10^{7}$ & $1.57 \times 10^{7}$ & 8352 & $44 \%$ \\
\hline Reco. upgoing $+\beta<1^{\circ}+\lambda>-5.2$ & 3058 & 358 & 2408 & $23 \%$
\end{tabular}

it was required for the estimated angular uncertainty on the muon-track direction $\beta$ that $\beta<1^{\circ}$. The second cut was applied on the track reconstruction quality parameter $\lambda$ and required $\lambda>-5.2$. This cut was calculated to maximize the discovery potential. After both cuts, $83 \%$ of the simulated signal events (assuming an $\mathrm{E}^{-2}$ spectrum) are reconstructed better than $1^{\circ}$. The median angular resolution is $0.46 \pm 0.1^{\circ}$. See figure 2. From the $3.94 \times 10^{8}$ events from data, 3058 events which were reconstructed as up-going remained after both cuts. $358 \pm 179$ atmospheric muons and $2408 \pm 722$ atmospheric neutrino were predicted by simulations. See table 1.

\subsection{Full sky search}

This search was performed with an unbinned maximum likelihood method, looking for an excess of events over the expected background of atmospheric muons and atmospheric neutrinos over the full sky visible to ANTARES. No significant excess of events could be found. The most significant cluster 

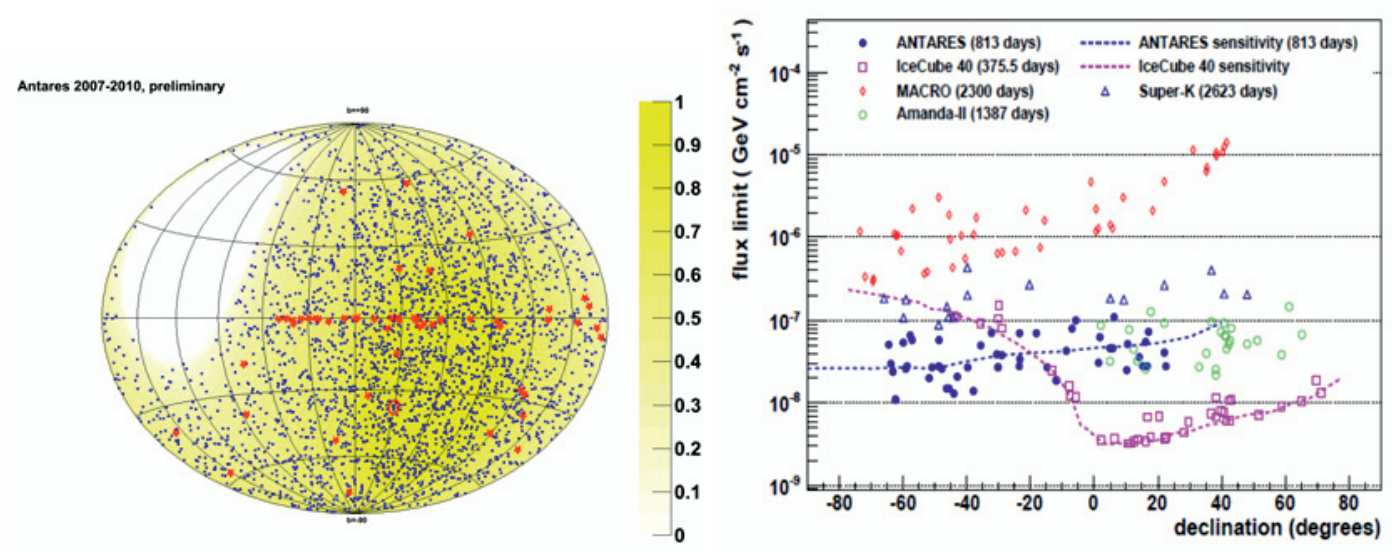

Figure 3. Left plot: Sky map, showing the acceptance of ANTARES (yellow area), the 3058 neutrino candidates (blue dots), the 51 candidate source (red stars) and the most significant cluster of events (red circle). Right plot: Upper limit ont the flux of candidate sources (markers) and the expected median sensitivities (dashed lines).

of events was found at $\alpha, \delta=\left(46.5^{\circ},-65.0^{\circ}\right)$, with 5 events within $1^{\circ}$ respectivly 9 events within $3^{\circ}$ of this position. The post-trial p-value for this cluster is $p=0.026(2.2 \sigma)[6]$.

\subsection{Candidate list search}

This search was performed on 51 potential neutrino sources, visible in the hard gamma-ray spectrum. No significant excess of neutrino events could be found. The most significant cluster of events was found for HESS $\mathrm{J} 1023-575$ at $\alpha, \delta=\left(155.83^{\circ},-57.76^{\circ}\right)$. The post-trial p-value for this source is $p=0.41[6]$. With this analysis, ANTARES has been able set the most stringent limits on the neutrino flux for most sources of the southern sky, as shown in figure 3 .

\section{Search for Gamma Ray Bursts}

ANTARES is connected to the Gamma Ray Burst Coordinate Network (GCN), in which the Swift and Fermi satellites are included. The GCN can alert ANTARES of the occurrence of a GRB in real-time. When a GRB alert is received, all raw data within two minutes after and up to one minute before the alert is stored. This data can be used for an extensive analysis in which also the position of the GRB (provided by the GCN) can be considered. The data from before the alert is received compensates for a delay in the alert and enables a search for a possible early neutrino signal (observable before the gamma ray signal). The advantage compared to the normal data taking, in which only a portion of to the total data reaching the shore station is used, is a gain in detection efficiency (shown in figure 4). The restriction of the search to the time window in which a GRB occurred results on a relatively low background of atmospheric muons and neutrinos.

A first analysis has been done for 37 selected GRBs recorded in 2007 (using only the 5 line period). No neutrino candidates in correlation with these GRBs could be observed. As shown in figure 4, ANTARES has been able to set upper limits on the fluence of muon-(anti)neutrinos from these GRBs for three different GRB models[7]. 

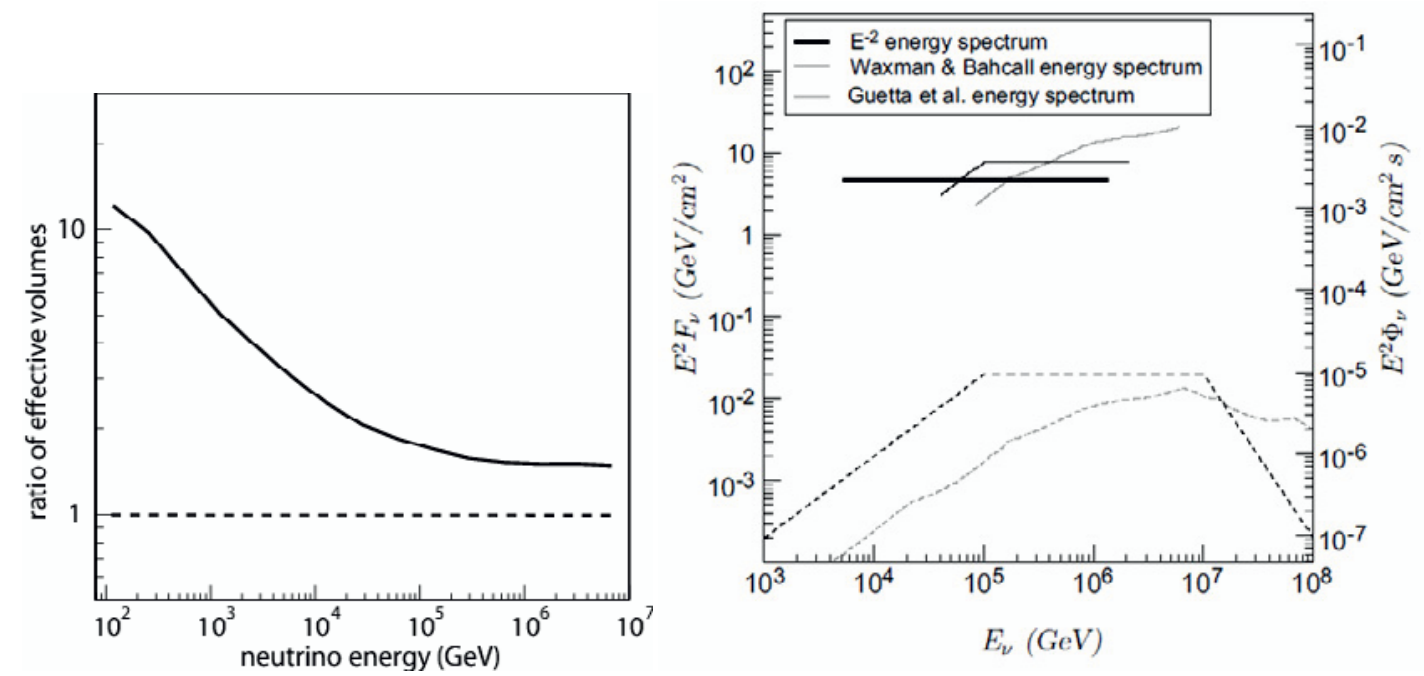

Figure 4. Left plot: Gain in detection efficiency (solid lines) for one GRB as a ratio of effective volume relative to the standard detection method (dashed line). Right plot: Upper limits on the muon-(anti)neutrino flux of the selected 37 GRBs (solid lines) for different energy spectra. The dashed lines show the total estimated muon(anti)neutrino fluence for the $37 \mathrm{GRBs}$ assuming according to Waxman\&Bahcall respectively Guetta et al. energy spectra. Both fluences include the effect of neutrino oscillations.

\section{Search for a diffuse flux}

From the observation of the diffuse flux of high energy cosmic rays, upper bounds for the diffuse neutrino flux have been derived[10, 11]. The diffuse neutrino flux is usually assumed to follow a $\mathrm{E}^{-2}$ spectrum. ANTARES has done a first search for the diffuse flux of high energy muon neutrinos. For this analysis, data taken between 12.2007 and 12.2009 was used. After basic quality cuts, data corresponding to a total of 334 days of data taking remained (136 days taken with 9 lines, 128 days with 10 lines and 70 days with 12 lines).

\subsection{Atmospheric muon rejection}

Simulations of the atmospheric muon background were done with the MUPAGE package. Two levels of cuts were used to reduce this background. The cuts are based on the number of lines which detected an event $\mathrm{N}_{1}$, the number of hits available for the track reconstruction $\mathrm{N}_{\text {hit }}$, the track reconstruction quality parameter $\lambda$ and the reconstructed zenith angle $\theta$. The first level required:

- $\mathrm{N}_{1}>1$

- $\mathrm{N}_{\text {hit }}>60$

- $\theta>100^{\circ}$

- $\lambda>-6$

The second level of cuts was derived from the events passing the first level and takes into account that in average $\lambda$ decreases with $\mathrm{N}_{\text {hit }}$. The second level required:

$$
\lambda=\left\{\begin{array}{cl}
-4.59-5.88 \times 10^{-3} & \text { if } N_{h i t} \leq 172 \\
-5.6 & \text { if } N_{h i t}>172
\end{array}\right.
$$




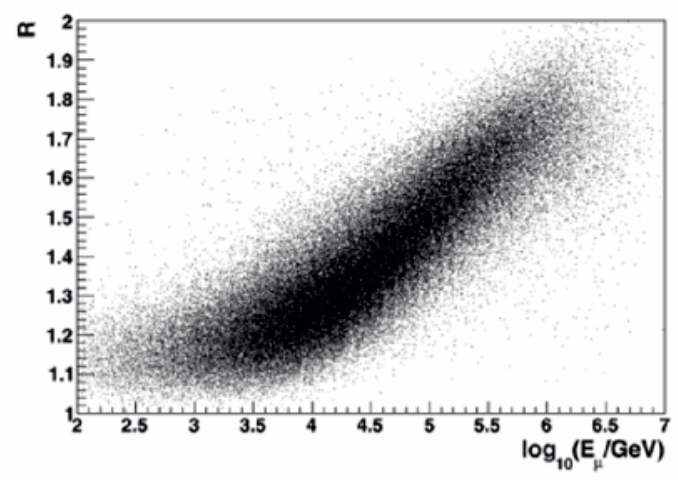

Figure 5. Distribution of the R paramter depending on the muon energy

After the second level, all of the simulated $2.2 \times 10^{8}$ atmospheric muon events, which were triggered and miss-reconstructed as up-going, were eliminated. From the originally $7.1 \times 10^{3}$ simulated atmospheric neutrinos, 116 remained[8].

\subsection{Atmospheric neutrino rejection}

The simulated atmospheric neutrinos for this analysis were weighted according to the Bartol parameterization. For the possible contribution of neutrinos from the decay of charmed mesons (prompt neutrinos) at energies above $10 \mathrm{TeV}$, the Recombination Quark Parton Model (RQPM) model was used (which gives the largest prompt neutrino flux from the models considered). Since the flux from astrophysical neutrino point sources is expected to follow a $\mathrm{E}^{-2}$ spectrum, while the flux of atmospheric neutrinos is expected to follow a softer $\mathrm{E}^{-3.7}$ spectrum (at high energies), the relative excess of astrophysical neutrinos should increase with energy. Therefore, a cut on an energy dependent parameter $\mathrm{R}$ was applied. For this, it is used that the chances for a muon of creating electromagnetic showers along a given path increase with the energy of the muon. These showers can emit light, which can be detected at the OMs later than the Cherenkov-Light emitted by the muon. Consequently, on average the mean number of these late hits over all OMs increases with energy. Thus, an energy dependent parameter can be defined as the mean number of hit repetitions within an event. With:

- $\mathrm{h}_{\mathrm{i}}:=$ the number of hits on a OM, which registered a hit that was used for the track reconstruction, within $500 \mathrm{~ns}$, beginning at the time at which the Cherenkov-Light belonging to the reconstructed track should have arrived at that $\mathrm{OM}$

- $\mathrm{N}:=$ the number of all OMs, which registered a hit that was used for the track reconstruction:

$\mathrm{R}$ is defined as:

$$
\mathrm{R}=\frac{1}{\mathrm{~N}} \cdot \sum \mathrm{h}_{\mathrm{i}}
$$

As shown in figure 5, there is a clear correlation between the $\mathrm{R}$ parameter and the muon energy. The optimal cut on $\mathrm{R}$ has been determined by minimizing the Model Rejection Factor[9]. For the purpose of finding the optimal cut on $\mathrm{R}$, the normalization of the the signal spectrum is irrelevant. The best cut has been found to be $\mathrm{R}>1.31$. With these cuts, $90 \%$ of the signal is expected in the energy range of $20 \mathrm{TeV}<\mathrm{E}_{v}<2.5 \mathrm{PeV}$. 

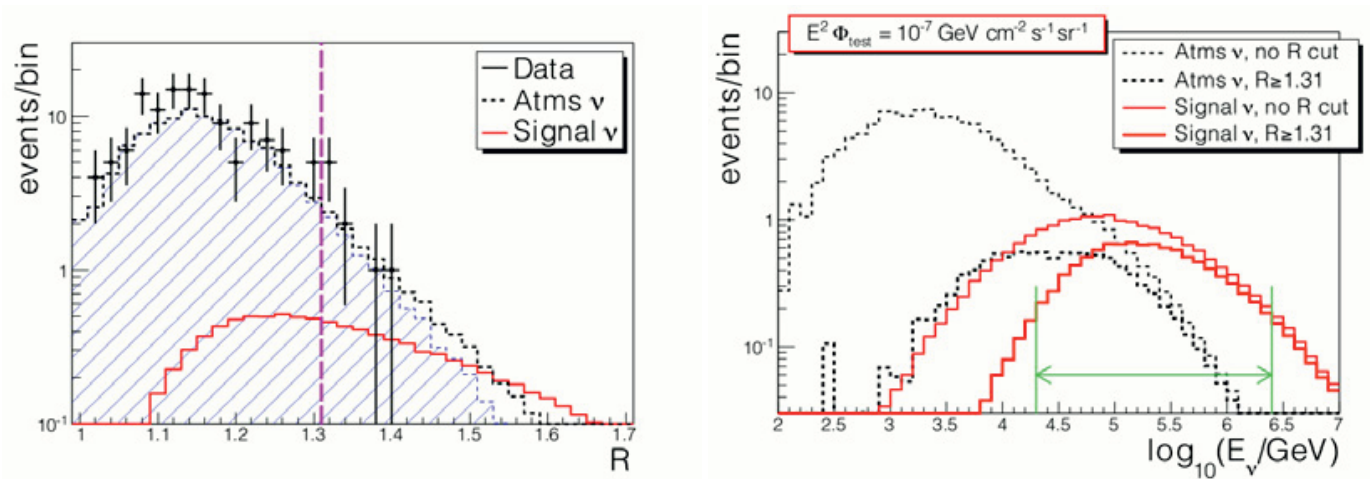

Figure 6. Left plot: Distribution of the expected observed atmospheric neutrino flux with the Bartol parameterization and the RQPM (black lines), an $\mathrm{E}^{-2}$ signal with arbitrary normalization (red lines) and the energy range where $90 \%$ of the signal is expected (green arrow). Right plot: Distribution of the R parameter for data (markers), atmospheric neutrinos with the Bartol parameterization (filled histogram), the prompt contribution according to the RQPM (black dashed line) and the signal at the level of the upper limit (solid red line). The vertical dashed line shows the cut at $R>1.31$.

\subsection{Results}

The expected background after all cuts is $n_{B}=10.7 \pm 2$ events. For the 334 days of data used for the analysis, after all cuts 9 events were observed. A 90\% CL upper limit on the diffuse neutrino flux could be set at $\mathrm{E}^{2} \Phi_{90 \%}=5.3 \times 10^{-8} \mathrm{GeVcm}^{-2} \mathrm{~s}^{-1} \mathrm{sr}^{-1}[8]$.

\section{Summary}

ANTARES has been continuously taking data since 2007. It has a broad physics program and runs analyses dedicated to answering important astrophysical questions such as, but not limited to, the origin of cosmic rays. Due to its location in the Mediterranean Sea, it complements the field of view of IceCube located at the South Pole. ANTARES has an excellent angular resolution, it has been shown that the telescope can cope with its background and the detector is well understood. With first analyses, competitive upper limits on the neutrino flux from point sources have been derived. 


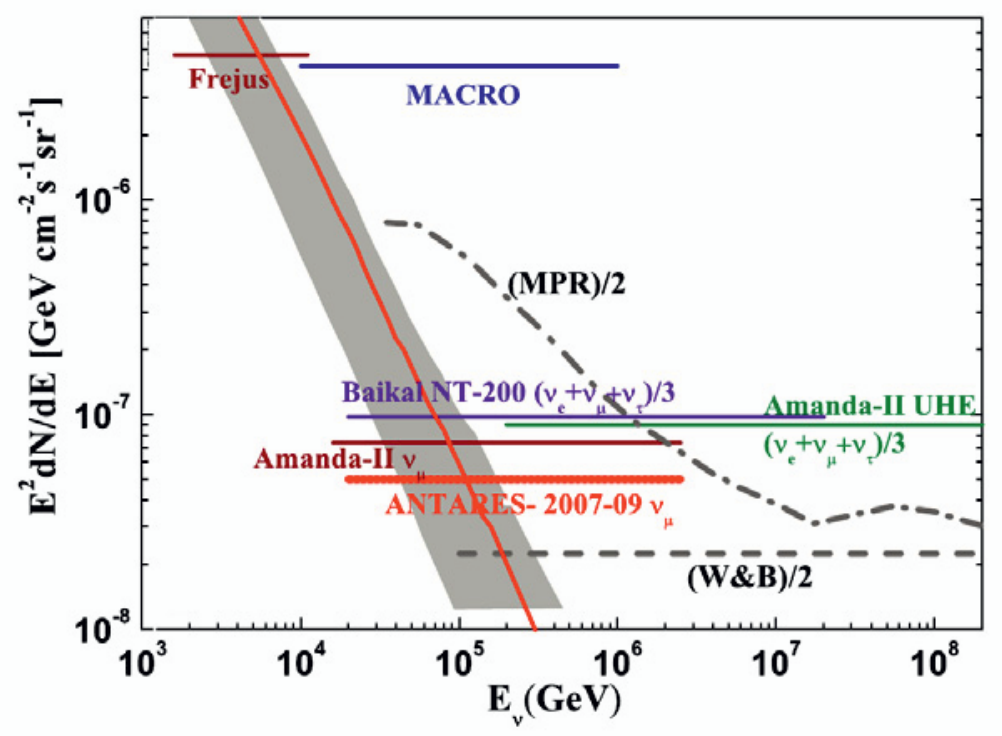

Figure 7. $90 \% \mathrm{CL}$ upper limit for an $\mathrm{E}^{-2}$ diffuse muon neutrino flux derived by ANTARES, compared to the upper limits derived by other experiments and the W\&B[10] and MPR[11] upper bounds (W\&B and MPR divided by two, to account for neutrino oscillations). 


\section{References}

[1] M. Ageron et al. (ANTARES Collaboration), ANTARES: the first undersea neutrino telescope, arXiv:1104.1607v1 [astro-ph.IM], 8 Apr 2011

[2] S Adrian-Martinez et al. (ANTARES Collaboration), The positioning system of the ANTARES Neutrino Telescope, 2012 JINST 7 T08002

[3] S. Escoffier (ANTARES Collaboration), The ANTARES detector: background sources and effects on detector performance, arXiv:0710.0527v1 [astro-ph], 2 Oct 2007

[4] Vivek Agrawal, T.K. Gaisser, Paolo Lipari, Todor Stanev, Atmospheric neutrino flux above 1 GeV, arXiv:hep-ph/9509423v1, 29 Sep 1995

[5] G. Carminati, M. Bazzotti, A. Margiotta and M. Spurio, Atmospheric MUons from PArametric formulas: a fast GEnerator for neutrino telescopes (MUPAGE), Comp. Phys. Comm. 179 (2008) 915

[6] S Adrian-Martinez et al. (ANTARES Collaboration), Search for Cosmic Neutrino Point Sources with Four Years of Data from the ANTARES Telescope, arXiv:1207.3105v2 [hep-ex], 21 Jul 2012

[7] S. Adrian-Martinez et al. (ANTARES Collaboration), Contributions to the 32nd International Cosmic Ray Conference (ICRC 2011) by the ANTARES collaboration, arXiv:1112.0478v1 [astroph.HE], 2 Dec 2011

[8] J.A. Aguilar et al. (ANTARES Collaboration), Search for a diffuse flux of high-energy $v_{\mu}$ with the ANTARES neutrino telescope, arXiv:1011.3772v1 [astro-ph.HE], 16 Nov 2010

[9] Gary C. Hill, Katherine Rawlins, Unbiased cut selection for optimal upper limits in neutrino detectors: the model rejection potential technique, arXiv:astro-ph/0209350v1, 18 Sep 2002

[10] E. Waxman and J. Bahcall, High energy neutrinos from astrophysical sources: An upper bound, Phys. Rev. D59 (1998) 023002; J. Bahcall, E.Waxman, High energy astrophysical neutrinos: The upper bound is robust, Phys. Rev. D64 (2001) 023002.

[11] K. Mannheim, R. J. Protheroe, J. P. Rachen, Cosmic ray bound for models of extragalactic neutrino production, Phys. Rev. D63 (2000) 023003. 